EGU2020-5740

https://doi.org/10.5194/egusphere-egu2020-5740

EGU General Assembly 2020

(c) Author(s) 2020. This work is distributed under

the Creative Commons Attribution 4.0 License.

\title{
Transformation of envelope solitons propagating over a bottom step
}

\author{
Alexey Slunyaev ${ }^{1,2,3}$, Guillaume Ducrozet ${ }^{4}$, and Yury Stepanyants ${ }^{3,5}$ \\ ${ }^{1}$ Instutute of Applied Physics, Department of Nonlinear Geophysical Processes, Nizhny Novgorod, Russia \\ (Slunyaev@appl.sci-nnov.ru) \\ ${ }^{2}$ National Research University-Higher School of Economics, Russia \\ ${ }^{3}$ Nizhny Novgorod State Technical University n.a. R.E. Alekseev, Nizhny Novgorod, Russia \\ ${ }^{4}$ Ecole Centrale Nantes, Nantes, France (Guillaume.Ducrozet@ec-nantes.fr) \\ ${ }^{5}$ University of Southern Queensland, Toowoomba, Australia (Yury.Stepanyants@usq.edu.au)
}

The problem of the weakly nonlinear wave transformation on a bottom step is studied analytically and numerically by means of the direct simulation of the Euler equation. It is assumed that the quasi-linear wave packets can be described by the nonlinear Schrödinger equation for surface waves in finite-depth water. The process of wave transformation in the vicinity of the bottom step can be described within the framework of the linear theory and the transformation coefficients (the transmission and reflection coefficients) can be determined by the approximate formula suggested in [1]. The fate of transmitted and reflected wave trains emerging from the incident envelope soliton can be determined with the help of the Inverse Scattering Technique $[2,3]$.

The parameters of secondary envelope solitons (their number, amplitudes, and speeds) asymptotically forming in the far-field zone are obtained analytically and compared against the numerically calculated ones, as the functions of the depth drop $h_{2} / h_{1}$, where $h_{1}$ and $h_{2}$ are the undisturbed water depths in front of and behind the bottom step, respectively. It is shown that the wave amplitudes can notably increase when the envelope soliton travels from the relatively shallow to much deeper water. The amplitudes of secondary solitons can exceed more than twice the amplitude of the incident wave.

The direct numerical simulation of envelope soliton transformation was undertaken by means of the High Order Spectral Method [4,5]. The comparison of approximate analytical solutions with the results of numerical simulations reveals the domains of very good agreement between the data where the approximate theory is applicable. In the meantime, the noticeable disagreement between the approximate nonlinear theory and the direct simulations is found when the theory is inapplicable.

The research by A.S. is supported by the RFBR grant No. 18-02-00042; he also acknowledges the support from the International Visitor Program of the University of Sydney and is grateful for the hospitality of the University of Southern Queensland. The research of Y.S. was support by the grant of the President of the Russian Federation for State support of scientific research of leading 
scientific Schools of the Russian Federation NSh-2485.2020.5.

[1] Kurkin, A.A., Semin, S.V., and Stepanyants, Yu.A., Transformation of Surface Waves over a Bottom Step. Izvestiya, Atmospheric and Oceanic Physics, 2015, Vol. 51, 214-223.

[2] Zakharov, V.E., Shabat, A.B., Exact theory of two-dimensional self-focussing and onedimensional self-modulation of waves in nonlinear media. Sov. Phys. JETP, 1972, Vol. 34, 62-69.

[3] Slunyaev, A., Klein, M., Clauss, G.F., Laboratory and numerical study of intense envelope solitons of water waves: generation, reflection from a wall and collisions. Physics of Fluids, 2017, Vol. 29, 047103.

[4] West, B.J., Brueckner, K.A., Janda, R.S., Milder, D.M., Milton, R.L., A new numerical method for surface hydrodynamics. J. Geophys. Res., 1987, Vol. 92, 11803-11824.

[5] Ducrozet, G., Gouin, M., Influence of varying bathymetry in rogue wave occurrence within unidirectional and directional sea-states. J. Ocean Eng. Mar. Energy, 2017, Vol. 3, 309-324. 